\title{
Dynamic rate-adaptive MIMO mode switching between spatial multiplexing and diversity
}

\author{
Chanhong Kim and Jungwoo Lee
}

\begin{abstract}
In this article, we propose a dynamic multiple-input multiple-output (MIMO) mode switching scheme between spatial multiplexing and diversity modes, which also includes adaptive modulation. At each transmission, we select the modulation level and the MIMO mode that maximize the spectral efficiency while satisfying a given target bit error rate. The dynamic MIMO mode scheme considers instantaneous spectral efficiency whereas the conventional static scheme considers only the average SNR. As for adaptive modulation, a new method is proposed to compute the SNR thresholds for adaptive modulation in each MIMO mode, and it can avoid the computational difficulty of the conventional Lagrangian (optimal) method at high average SNR. To deal with the case where the rates of the two MIMO modes are the same, we also propose a new measure based on the BER exponent, which has lower computational complexity than a conventional measure. Numerical results show that the proposed dynamic mode switching improves over the conventional static mode switching in terms of average spectral efficiency.
\end{abstract}

\section{Introduction}

Today's wireless communication systems demand high data rate and spectral efficiency with increased reliability. Multiple-input multiple-output (MIMO) systems have been popular techniques to achieve these goals because increased data rate is possible through spatial multiplexing scheme [1] or improved diversity order is possible through transmit diversity scheme (e.g., space-time block code, STBC) [2]. Other ways are link adaptation techniques, where transmission parameters such as modulation and coding are dynamically adapted to the varying channel condition [3]. A typical link adaptation technique is adaptive modulation in which an adequate modulation level is selected by means of the current signal-to-noise ratio (SNR).

Recently, adaptive modulation schemes in conjunction with MIMO techniques have been investigated [4-11]. The prior study in the literature mainly tried to maximize the average spectral efficiency (ASE) for only one MIMO mode, either spatial multiplexing [8] or transmit diversity $[5-7,11]$. In [12], the mode switching between diversity and multiplexing was first proposed. But the authors focused on the situation where both MIMO

*Correspondence: junglee@snu.ac.kr

School of Electrical Engineering and Computer Science, Seoul National University, Seoul, Korea modes have equal spectral efficiency without considering adaptive modulation, so that they showed the result that spatial multiplexing is preferred in low SNR region. The mode switching scheme combined with adaptive modulation was proposed in $[9,10]$, but the analysis was focused on the static mode switching which depends only on the average SNR.

In this article, we propose a dynamic MIMO mode switching scheme which considers instantaneous channel condition in conjunction with rate adaptation. Although the adaptive modulation part is based on the existing methods [5,7-9,11], we compare the performance of the existing techniques, and also propose a sub-optimal method to obtain the SNR thresholds for the average BER constraint. Its complexity is lower than that of the optimal method using a Lagrange multiplier, but the performance degradation is negligible. The proposed mode switching scheme is based on the instantaneous spectral efficiency (ISE). In case the ISE's of the two modes are equal, an additional rule is necessary for mode selection. Although the Demmel condition number proposed in [12] can be a choice, we propose a new method which has lower complexity than the Demmel condition number without performance loss.

This article is organized as follows. In the section of System overview, we outline the system and the channel model as well as the structure of the considered

\section{Springer}

(C) 2012 Kim and Lee; licensee Springer. This is an Open Access article distributed under the terms of the Creative Commons Attribution License (http://creativecommons.org/licenses/by/2.0), which permits unrestricted use, distribution, and reproduction in any medium, provided the original work is properly cited. 
MIMO mode switching system. The dynamic MIMO mode switching scheme combined with adaptive modulation is then proposed in the section of rate-adaptive MIMO mode switching. In the section of Simulation results, we compare the performance of the proposed algorithm with that of the existing methods. Since numerical methods are necessary in order to get the SNR thresholds for the average BER constraint, we shows detailed results in this section. Finally, conclusions are drawn in the last section.

\section{System overview}

We consider a MIMO system with $M$ transmit antennas and $N$ receive antennas. The block diagram of the proposed system is shown in Figure 1. The system consists of a transmitter with a switch between a multiplexing and a diversity modulator, a receiver unit with the corresponding pair of receivers, a modulation level and mode selector, and a low rate feedback path. At the receiver side, the modulation level and the MIMO mode are selected according to the current channel condition. The information about the selected modulation level and the MIMO mode is sent to the transmitter through the feedback path. The transmitter then switches the MIMO mode with the modulation level based on the feedback information.

Suppose that the $N \times M$ flat fading channel matrix $\mathbf{H}$ has i.i.d. complex Gaussian random entries. The $(i, j)$ th entry $[\mathbf{H}]_{i, j}=h_{i j}$ is distributed as $\mathcal{C N}(0,1)^{\mathrm{a}}$. The channel is assumed to be quasi-static (channel coefficients do not change during one time interval, and change independently in the next interval). The input-output relation for the MIMO channel is given by

$$
\mathbf{y}=\sqrt{\frac{E_{s}}{M}} \mathbf{H s}+\mathbf{n},
$$

where $y$ is the $N \times 1$ received signal vector, $E_{s}$ is the average energy per symbol, $\mathbf{s}$ is the transmitted signal vector with energy $M$, i.e., $\mathcal{E}\left[\mathbf{s}^{H} \mathbf{s}\right]=M^{\mathrm{b}}$, and $\mathbf{n}$ is an $N \times 1$ i.i.d. complex additive white Gaussian noise (AWGN) vector with the distribution $\mathcal{C N}\left(0, N_{0} \mathbf{I}_{N}\right)$. Let $\rho$ be the average SNR at the receiver, which is given by $\rho=\frac{E_{s}}{N_{0}}$. We have omitted the time index in (1) for convenience. We also assume perfect channel knowledge at the receiver and zero feedback delay.

\section{Rate-adaptive MIMO mode switching}

In this article, we propose a new rate-adaptive MIMO mode switching algorithm. The goal is to maximize the ASE while satisfying a given bit error ratio constraint. The proposed algorithm can be summarized by the following three steps.

1. Calculate the post-processing SNR in each MIMO mode.

2. Decide the modulation order in each MIMO mode.

3. Decide one MIMO mode based on a given selection rule.

For analysis, we consider a linear receiver for the spatial multiplexing mode, and orthogonal space-time block codes (OSTBC) for the diversity mode. In Step 2, we analyze several adaptive modulation techniques subject to an instantaneous BER constraint as well as an average one. In Step 3, we propose the mode selection rule based on the ISE as well as the rule which can be applied to the case when both of the two MIMO modes have the same data rate.

\section{Post-processing SNR calculation (Step 1)}

The post-processing SNR at the receiver is calculated separately in each MIMO mode with a given detection algorithm. At first, in the spatial multiplexing mode, the post-processing SNR of the $m$ th $(m=1,2, \ldots, M)$ output data stream of the zero forcing (ZF) receiver, denoted as $\gamma_{m, \mathrm{ZF}}$, is given by ([13], Eq. (7.43))

$$
\gamma_{m, \mathrm{ZF}}=\frac{\rho}{M} \frac{1}{\left[\left(\mathbf{H}^{H} \mathbf{H}\right)^{-1}\right]_{m, m}},
$$

and the SNR of the minimum mean-square error (MMSE) receiver, denoted as $\gamma_{m, \mathrm{MMSE}}$, is given by ([13], Eq. (7.49))

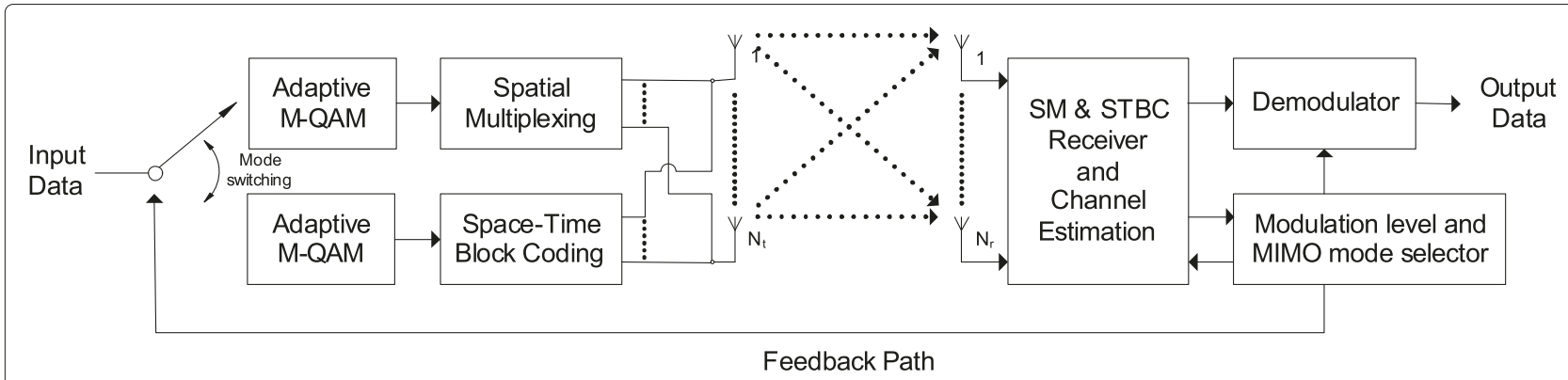

Figure 1 Block diagram of MIMO mode switching scheme combined with adaptive modulation. 


$$
\gamma_{m, \mathrm{MMSE}}=\frac{1}{\left[\left(\frac{\rho}{M} \mathbf{H}^{H} \mathbf{H}+\mathbf{I}_{M}\right)^{-1}\right]_{m, m}}-1 .
$$

The post-processing SNR of the OSTBC system, denoted as $\gamma_{\text {оствC }}$, is given by

$$
\gamma_{\text {OSTBC }}=\frac{\rho}{\zeta M}\|\mathbf{H}\|_{F}^{2}=\frac{\rho}{\zeta M} \sum_{i=1}^{N} \sum_{j=1}^{M}\left|h_{i, j}\right|^{2},
$$

where $\zeta$ is the code rate of the OSTBC.

\section{Decision of the modulation order (Step 2)}

Using the post-processing SNR obtained in Step 1, we can choose an appropriate modulation order for each MIMO mode which enhances spectral efficiency without exceeding a given target BER at the receiver. Since adaptive modulation for one MIMO mode with a given BER constraint has been studied in $[4-8,11]$, we take a similar approach of the literature. For analysis, we consider a discrete rate adaptive system for which the constellations are restricted to a finite set $\mathcal{M}=\left\{M_{0}, M_{1}, \ldots, M_{L}\right\}$ with Gray coded quadrature amplitude modulation (QAM), where $M_{l}$ denotes the constellation size and $M_{l-1}<$ $M_{l}, \forall l$. The SNR range is subdivided into $L+1$ bins bounded by the switching threshold $\theta_{l}(l=0,1, \ldots, L+$ $1)$ where $\theta_{0}=0$. Let $\gamma$ be the post-processing SNR. The receiver chooses the constellation $M_{l}$ whenever $\theta_{l} \leq$ $\gamma<\theta_{l+1}$. If $\gamma<\theta_{1}$, data transmission is suspended for the corresponding channel since the respective BER constraint cannot be satisfied. Moreover, the maximum SNR threshold is set to infinity, i.e., $\theta_{L+1}=\infty$.

\section{SNR thresholds for instantaneous BER constraint}

An easy way to set the switching thresholds $\theta_{l}$ 's is to use the instantaneous BER (I-BER). In this approach, the BER of every reception has to be less than or equal to the target BER $\delta_{0}$. In order to meet the constraint, the BER for a QAM in AWGN channels can be used. Although the exact BER expressions for M-QAM are shown in [14], they are not easily inverted with respect to the SNR, so that a numerical method is necessary. Instead, in the adaptive modulation literature [5-8], an exponential function form is used, which is given by

$$
P_{e}\left(\gamma, M_{l}\right) \approx a_{l} \exp \left(-c_{l} \gamma\right)
$$

where $a_{l}=0.2$ and $c_{l}$ is a constellation specific constant defined as [4]

$$
c_{l}=\left\{\begin{array}{ll}
\frac{6}{5 \cdot 2^{l}-4} & \text { for rectangular QAM (odd } l) \\
\frac{3}{2\left(2^{l}-1\right)} & \text { for square QAM (even } l)
\end{array} .\right.
$$

If we want a more accurate form than the above approximation, we can find the modulation specific constants $a_{l}$ and $c_{l}$ numerically using curve-fitting methods [11]. Table 1 shows those values of M-QAM's which are used
Table 1 Constellation specific constants for BER approximation in AWGN channels [11]

\begin{tabular}{ccccc}
\hline Modulation & BPSK & QPSK & 16-QAM & 64-QAM \\
\hline$a_{l}$ & 0.1978 & 0.1853 & 0.1613 & 0.1351 \\
$c_{l}$ & 1.0923 & 0.5397 & 0.1110 & 0.0270 \\
\hline
\end{tabular}

in [11]. Inverting (5) with respect to $\gamma$, the switching threshold is determined as

$$
\theta_{l}=\frac{1}{c_{l}} \ln \left(\frac{a_{l}}{\delta_{0}}\right) \text {. }
$$

Although it is simple, I-BER approach keeps the instantaneous BER at all time instants below the target BER $\delta_{0}$. This is so conservative that the average BER (A-BER) is lower than $\delta_{0}$. In order to make the A-BER be equal to $\delta_{0}$, SNR thresholds should be lowered. Therefore, there is potential for improving the ASE by adjusting the switching threshold of each modulation.

\section{SNR thresholds for average BER constraint}

Generally, the ASE $\eta$ for one channel use is given by

$$
\eta=\sum_{l=1}^{L} b_{l} \cdot p_{l}
$$

where $b_{l}=\log _{2} M_{l}$ is the number of bits corresponding to the $l$ th modulation and $p_{l}$ is the probability that the postprocessing SNR falls into the $l$ th bin, given by

$$
p_{l}=\int_{\theta_{l}}^{\theta_{l+1}} f(\gamma) d \gamma
$$

where $f(\gamma)$ is the probability density function (pdf) of the post-processing SNR. The A-BER can be denoted as the average number of error bits $N_{e, \text { avg }}$ divided by the average number of transmitted bits $N_{b, a v g}$. It is observed that $N_{b, \text { avg }}=\eta$ by the definition in (8) and $N_{e, \text { avg }}$ is given by

$$
N_{e, \mathrm{a} v g}=\sum_{l=1}^{L} b_{l} \cdot P_{e}(l),
$$

where $P_{e}(l)$ is the A-BER when the SNR falls into the $l$ th bin, given by

$$
P_{e}(l)=\int_{\theta_{l}}^{\theta_{l+1}} P_{e}\left(\gamma, M_{l}\right) f(\gamma) d \gamma .
$$

Since it has already been known that the pdf's of the two MIMO modes have Gamma distributions, using the above formulas, the ASE and the A-BER can be obtained as closed forms as (26) and (30) in case of ZF spatial multiplexing system and (34) and (38) in case of OSTBC system, respectively. See Appendix for details. The pdf of $\gamma_{m \text {,MMSE }}$ can be well approximated to a Gamma distribution and to 
a generalized Gamma distribution [15]. Thus, though it is approximation, the analysis of the MMSE receiver can be done with the same procedure as that of the $\mathrm{ZF}$ receiver.

Optimal method In A-BER approach, the goal is to maximize the ASE under the constraint that the A-BER should be lower than or equal to $\delta_{0}$. Defining the set of adjustable switching thresholds as $\Theta=\left\{\theta_{l} \mid l=1,2, \ldots, L\right\}^{\mathrm{c}}$, the optimization problem can be formulated as

$$
\Theta_{o}=\underset{\Theta}{\arg \max } \eta \text {, subject to } P_{e, \mathrm{avg}} \leq \delta_{0} .
$$

This problem can be solved with a Lagrange multiplier. Since $P_{e, \text { avg }}$ is denoted as

$$
P_{e, \mathrm{avg}}=\frac{N_{e, \mathrm{avg}}}{N_{b, \mathrm{avg}}}=\frac{N_{e, \mathrm{a} v g}}{\eta},
$$

changing the constraint $P_{e, \text { avg }} \leq \delta_{0}$ into $N_{e, \text { avg }} \leq \delta_{0} \eta$ for convenience, the Lagrangian of (12) is defined as

$$
\mathcal{L}(\Theta, \lambda)=\eta+\lambda\left(N_{e, \mathrm{avg}}-\delta_{0} \eta\right) .
$$

Differentiating (14) with respect to $\theta_{l}$ and equating to zero, the following relationship for $l=2,3, \ldots, L$ is obtained as

$$
\lambda=\frac{b_{l}-b_{l-1}}{b_{l-1} P_{e}\left(\theta_{l}, M_{l-1}\right)-b_{l} P_{e}\left(\theta_{l}, M_{l}\right)-\delta_{0}\left(b_{l-1}-b_{l}\right)} .
$$

According to the relationship, once $\theta_{1}$ is chosen, all the other $\theta_{l}$ 's are uniquely determined. Thus, the optimal SNR thresholds can be found numerically by adjusting $\theta_{1}$ only.

Descending search method (sub-optimal) Intuitively, as the SNR range assigned to a high order modulation increases, the ASE increases while the BER also increases at the same time. Thus, in order to maximize the ASE, the SNR threshold $\theta_{l}$ in descending order $(l=L, L-1, \ldots, 1)$ has to be lowered as much as possible. Since the A-BER has to be kept below $\delta_{0}$, we take the constraint that $P_{e}(l) \leq$ $\delta_{0}, \forall l$. The detailed algorithm is as follows:

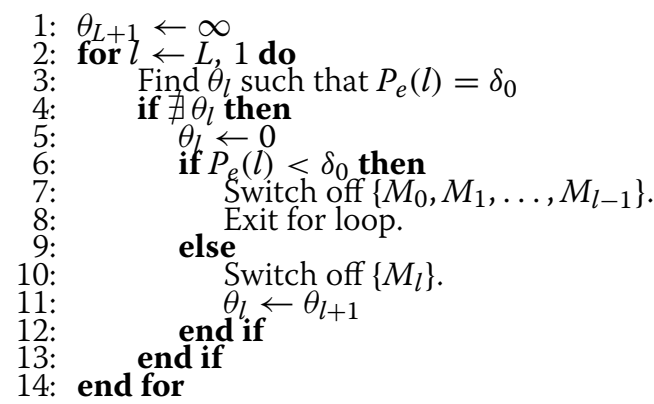

In the proposed algorithm, a certain modulation can be completely switched off because the solution that satisfies
$P_{e}(l)=\delta_{0}$ may not exist at all or only higher order modulations may satisfy the BER constraint on the whole SNR region.

Although the A-BER approach has higher computational complexity than the I-BER approach, the thresholds can be calculated off-line, and the A-BER approach is still practical.

\section{Dynamic MIMO mode switching (Step 3)}

After the modulation order in each MIMO mode is chosen, the ISE can be calculated. The ISE of the spatial multiplexing system with ZF receiver, denoted as $R_{\mathrm{ZF}}$, can be written as

$$
R_{\mathrm{ZF}}=\sum_{m=1}^{M} b_{m, \mathrm{ZF}} \quad \text { (bits/channel use), }
$$

where $b_{m, \mathrm{ZF}}$ is the number of bits corresponding to the selected modulation on the $m$ th subchannel. Likewise, the ISE of the OSTBC system, denoted as $R_{\text {OSTBC }}$, can be expressed as

$$
R_{\mathrm{OSTBC}}=\zeta \cdot b_{\mathrm{OSTBC}} \quad \text { (bits/channel use), }
$$

where $b_{\text {OSTBC }}$ denotes the number of bits corresponding to the selected modulation.

If $R_{\mathrm{ZF}}$ and $R_{\mathrm{OSTBC}}$ are different from each other, the mode selection rule is as follows: if $R_{\mathrm{ZF}}>R_{\mathrm{OSTBC}}$, spatial multiplexing is chosen for the next transmission mode, and vice versa. In case of $R_{\mathrm{ZF}}=R_{\mathrm{OSTBC}}$, a general rule is to select a mode that gives lower BER, and the MIMO mode can be chosen based on the following two methods. One method is the Demmel condition number approach which was proposed in [12]. The Demmel condition number $\kappa_{D}$ is defined as

$$
\kappa_{D}:=\frac{\|\mathbf{H}\|_{F}}{\lambda_{\min }(\mathbf{H})},
$$

where $\lambda_{\min }(\mathbf{H})$ denotes the minimum singular value of $\mathbf{H}$, and spatial multiplexing is preferred if

$$
\kappa_{D} \leq \frac{d_{\text {min,ZF }}}{d_{\text {min,OSTBC }}},
$$

where $d_{\text {min,ZF }}$ is the minimum Euclidean distance of the transmit constellation of the spatial multiplexing system with a ZF receiver, and $d_{\text {min,OSTBC }}$ is the minimum Euclidean distance of the OSTBC system.

Another method is to use the I-BER of each MIMO mode, which can be measured by the exponent of the BER Equation (5). In other words, a MIMO mode which has lower I-BER than the other can be chosen. Assuming that the approximation of the I-BER in (5) and the constellation specific constants in [4] are used, the following rule can be derived. Spatial multiplexing is preferred if

$$
c_{\min , \mathrm{ZF}} \gamma_{\min , \mathrm{ZF}} \geq c_{\mathrm{OSTBC}} \gamma_{\mathrm{OSTBC}},
$$


where $\gamma_{\text {min,ZF }}=\min _{m} \gamma_{m, \mathrm{ZF}}$, which means that the worst stream of the spatial multiplexing mode is used to calculate the I-BER. Although the Demmel condition number can be used for mode switching, it has higher complexity than (20) because singular value decomposition is necessary to get $\kappa_{D}$ in (18), whereas (20) only uses the parameters which are already obtained from the previous steps. Therefore, the proposed measure is desirable in terms of computational complexity.

\section{Simulation results}

In simulation, a simple MIMO system is considered whose antenna configuration is $M=N=2$. Constellations are restricted to $\mathrm{M}$-QAM with $\mathcal{M}=\{1,2,4,16,64\}$, where 1 means no transmission, 2 BPSK, 4 QPSK, and so on. The target BER $\delta_{0}$ is set to be $10^{-3}$. The ASE and the ABER are averaged over $10^{5}$ channel realizations under a block fading channel model, where the entries of channel matrix do not change during the transmission of two vector symbols.

\section{Adaptive modulation in a MIMO mode SNR thresholds for the I-BER constraint}

The thresholds are obtained in the following three ways. In the first method, the thresholds are obtained from numerical search using the exact BER expressions of $M$ QAM in AWGN channels. In the second method, the thresholds are from (7) with constellation specific constants defined in. In the third method, the thresholds are also from (7) with the constants of $a_{l}=0.2$ and $c_{l}$ defined in (6). As shown in Table 2, the second thresholds are quite close to the first, so the first and the third thresholds are used for the I-BER simulation.

\section{SNR thresholds for the A-BER constraint}

It is observed that, in (30) and (38) of Appendix, the A-BER of each MIMO mode depends on the three parameters, $M, N$, and $\rho$. Thus, if $M$ and $N$ are fixed, the optimal SNR thresholds change only according to $\rho$. We obtained the optimal thresholds using a Lagrange multiplier, and obtained the sub-optimal thresholds using the descending search method described in the previous section by changing $\rho$ from 0 to $40 \mathrm{~dB}$ with the interval of $1 \mathrm{~dB}$. Tables 3 and 4 show the optimal and the sub-optimal thresholds of the spatial multiplexing system with ZF receiver, and Tables 5 and 6 show those

Table 2 SNR thresholds for the I-BER constraint $\left(\delta_{0}=10^{-3}\right)$

\begin{tabular}{ccccc}
\hline Modulation & BPSK & QPSK & 16-QAM & 64-QAM \\
\hline Exact BER [14] & 6.79 & 9.80 & 16.54 & 22.55 \\
Huang's method [11] & 6.85 & 9.86 & 16.61 & 22.59 \\
Zhou's method [4] & 7.24 & 10.25 & 17.24 & 23.47 \\
\hline
\end{tabular}

Table 3 SNR thresholds for the spatial multiplexing system with ZF receiver from lagrange multiplier method $\left(M=N=2, \delta_{0}=10^{-3}\right)$

\begin{tabular}{|c|c|c|c|c|}
\hline Average SNR & BPSK & QPSK & 16-QAM & 64-QAM \\
\hline 0 & 6.48 & 10.07 & 16.83 & 23.15 \\
\hline 1 & 6.40 & 10.00 & 16.75 & 23.08 \\
\hline 2 & 6.31 & 9.92 & 16.67 & 23.00 \\
\hline 3 & 6.20 & 9.82 & 16.57 & 22.91 \\
\hline 4 & 6.09 & 9.72 & 16.47 & 22.81 \\
\hline 5 & 5.96 & 9.61 & 16.36 & 22.70 \\
\hline 6 & 5.82 & 9.49 & 16.23 & 22.58 \\
\hline 7 & 5.68 & 9.37 & 16.11 & 22.46 \\
\hline 8 & 5.54 & 9.24 & 15.98 & 22.34 \\
\hline 9 & 5.38 & 9.11 & 15.84 & 22.21 \\
\hline 10 & 5.21 & 8.96 & 15.69 & 22.06 \\
\hline 11 & 5.03 & 8.80 & 15.53 & 21.91 \\
\hline 12 & 4.88 & 8.68 & 15.40 & 21.79 \\
\hline 13 & 4.76 & 8.57 & 15.30 & 21.69 \\
\hline 14 & 4.67 & 8.50 & 15.22 & 21.62 \\
\hline 15 & 4.61 & 8.45 & 15.17 & 21.57 \\
\hline 16 & 4.54 & 8.39 & 15.11 & 21.51 \\
\hline 17 & 4.46 & 8.32 & 15.04 & 21.45 \\
\hline 18 & 4.37 & 8.24 & 14.96 & 21.37 \\
\hline 19 & 4.29 & 8.18 & 14.89 & 21.31 \\
\hline 20 & 4.21 & 8.11 & 14.82 & 21.25 \\
\hline 21 & 4.14 & 8.05 & 14.76 & 21.19 \\
\hline 22 & 4.06 & 7.98 & 14.69 & 21.13 \\
\hline 23 & 3.95 & 7.89 & 14.60 & 21.04 \\
\hline 24 & 3.81 & 7.78 & 14.48 & 20.93 \\
\hline 25 & 3.63 & 7.63 & 14.33 & 20.79 \\
\hline 26 & 3.40 & 7.44 & 14.14 & 20.61 \\
\hline 27 & 3.12 & 7.21 & 13.91 & 20.40 \\
\hline 28 & 2.78 & 6.93 & 13.63 & 20.14 \\
\hline 29 & 2.39 & 6.62 & 13.32 & 19.85 \\
\hline 30 & 1.93 & 6.25 & 12.96 & 19.53 \\
\hline 31 & 1.39 & 5.83 & 12.54 & 19.15 \\
\hline 32 & 0.76 & 5.34 & 12.07 & 18.73 \\
\hline$\geq 33$ & N. A. & N. A. & N. A. & N. A. \\
\hline
\end{tabular}

of the OSTBC system. We used (5) with constellation specific constants defined in as a BER function in AWGN channels. In the table, 'N. A.' means 'not available' and ' $x$ ' means that the corresponding modulation is completely switched off. As shown in Tables 3 and 5, searching for the optimal SNR thresholds fails at high average SNR. This is because lower modulation orders cannot be switched off even when using only one higher 
Table 4 SNR thresholds for the spatial multiplexing system with ZF receiver from descending search

$\operatorname{method}\left(M=N=2, \delta_{0}=10^{-3}\right)$

\begin{tabular}{|c|c|c|c|c|}
\hline Average SNR & BPSK & QPSK & 16-QAM & 64-QAM \\
\hline 0 & 6.48 & 9.65 & 16.56 & 22.58 \\
\hline 1 & 6.40 & 9.61 & 16.55 & 22.58 \\
\hline 2 & 6.31 & 9.55 & 16.54 & 22.57 \\
\hline 3 & 6.21 & 9.48 & 16.52 & 22.57 \\
\hline 4 & 6.10 & 9.40 & 16.50 & 22.56 \\
\hline 5 & 5.99 & 9.31 & 16.47 & 22.56 \\
\hline 6 & 5.90 & 9.20 & 16.43 & 22.55 \\
\hline 7 & 5.82 & 9.08 & 16.39 & 22.54 \\
\hline 8 & 5.76 & 8.94 & 16.34 & 22.52 \\
\hline 9 & 5.73 & 8.78 & 16.28 & 22.50 \\
\hline 10 & 5.74 & 8.61 & 16.21 & 22.48 \\
\hline 11 & 5.78 & 8.41 & 16.13 & 22.45 \\
\hline 12 & 5.85 & 8.21 & 16.03 & 22.42 \\
\hline 13 & 5.95 & 8.00 & 15.92 & 22.38 \\
\hline 14 & 6.07 & 7.80 & 15.79 & 22.33 \\
\hline 15 & 6.19 & 7.62 & 15.64 & 22.27 \\
\hline 16 & 6.30 & 7.47 & 15.48 & 22.20 \\
\hline 17 & 6.39 & 7.35 & 15.30 & 22.11 \\
\hline 18 & 6.46 & 7.28 & 15.11 & 22.01 \\
\hline 19 & 6.49 & 7.23 & 14.91 & 21.90 \\
\hline 20 & 6.51 & 7.21 & 14.73 & 21.76 \\
\hline 21 & 6.51 & 7.21 & 14.57 & 21.61 \\
\hline 22 & 6.51 & 7.21 & 14.43 & 21.44 \\
\hline 23 & 6.51 & 7.21 & 14.34 & 21.25 \\
\hline 24 & 6.51 & 7.21 & 14.29 & 21.03 \\
\hline 25 & 6.53 & 7.19 & 14.27 & 20.80 \\
\hline 26 & 6.56 & 7.16 & 14.30 & 20.53 \\
\hline 27 & 6.60 & 7.11 & 14.37 & 20.24 \\
\hline 28 & 6.66 & 7.05 & 14.48 & 19.92 \\
\hline 29 & 6.73 & 6.97 & 14.62 & 19.57 \\
\hline 30 & 6.82 & 6.87 & 14.80 & 19.17 \\
\hline 31 & $\times$ & 6.76 & 15.03 & 18.73 \\
\hline 32 & $x$ & 6.26 & 15.33 & 18.23 \\
\hline 33 & $\times$ & 6.41 & 15.71 & 17.66 \\
\hline 34 & $\times$ & 6.13 & 16.24 & 17.00 \\
\hline 35 & $\times$ & 6.14 & $\times$ & 16.21 \\
\hline 36 & $x$ & 6.63 & $x$ & 15.24 \\
\hline 37 & 6.48 & 7.24 & $x$ & 13.99 \\
\hline 38 & 5.79 & 8.14 & $\times$ & 12.21 \\
\hline 39 & 5.18 & $x$ & $\times$ & 9.14 \\
\hline$\geq 40$ & $\times$ & $x$ & $\times$ & $-\infty$ \\
\hline
\end{tabular}

Table 5 SNR thresholds for OSTBC system obtained from Lagrange multiplier method $\left(M=N=2, \delta_{0}=10^{-3}\right)$

\begin{tabular}{|c|c|c|c|c|}
\hline Average SNR & BPSK & QPSK & 16-QAM & 64-QAM \\
\hline 0 & 6.36 & 9.96 & 16.72 & 23.04 \\
\hline 1 & 6.22 & 9.84 & 16.59 & 22.92 \\
\hline 2 & 6.03 & 9.67 & 16.42 & 22.76 \\
\hline 3 & 5.80 & 9.47 & 16.21 & 22.56 \\
\hline 4 & 5.56 & 9.26 & 16.00 & 22.36 \\
\hline 5 & 5.32 & 9.05 & 15.79 & 22.16 \\
\hline 6 & 5.03 & 8.80 & 15.53 & 21.91 \\
\hline 7 & 4.63 & 8.46 & 15.18 & 21.59 \\
\hline 8 & 4.17 & 8.08 & 14.79 & 21.21 \\
\hline 9 & 4.08 & 8.00 & 14.71 & 21.14 \\
\hline 10 & 4.26 & 8.15 & 14.86 & 21.29 \\
\hline 11 & 4.47 & 8.33 & 15.04 & 21.46 \\
\hline 12 & 4.57 & 8.41 & 15.13 & 21.54 \\
\hline 13 & 4.49 & 8.35 & 15.06 & 21.47 \\
\hline 14 & 4.19 & 8.09 & 14.80 & 21.23 \\
\hline 15 & 3.86 & 7.82 & 14.52 & 20.97 \\
\hline 16 & 3.81 & 7.78 & 14.48 & 20.93 \\
\hline 17 & 3.90 & 7.85 & 14.56 & 21.00 \\
\hline 18 & 3.96 & 7.90 & 14.61 & 21.05 \\
\hline 19 & 3.86 & 7.82 & 14.52 & 20.97 \\
\hline 20 & 3.49 & 7.51 & 14.21 & 20.68 \\
\hline 21 & 2.62 & 6.81 & 13.50 & 20.02 \\
\hline 22 & 0.19 & 4.90 & 11.67 & 18.37 \\
\hline$\geq 23$ & N. A. & N. A. & N. A. & N. A. \\
\hline
\end{tabular}

order modulation for the whole SNR region produces the A-BER below $\delta_{0}$, and also because the curve fitting approximation of the BER is used [11]. Note that it is assumed that all the constellations are used in the optimal method. In [11], the last valid thresholds with a lower SNR is used to tackle this situation, but it causes some performance loss. Instead, in the suboptimal method, since it is possible to switch off some constellations, the thresholds can always be obtained. Note that only 64-QAM is used for the entire instantaneous SNR region with high average SNR as shown in Tables 4 and 6.

\section{ASE and BER performance}

Figures 2 and 3 shows the ASE and the A-BER of the spatial multiplexing system with ZF receiver, and Figures 4 and 5 show those of the OSTBC system. Note that ' $\mathrm{Z}$ ', 'H' 'LM', and 'DS' stand for the Zhou method [4], the Huang method [11], the Lagrange multiplier method, and the descending search method, respectively. The Zhou method means that the constellation specific constants 
Table 6 SNR thresholds for OSTBC system obtained from descending search method $\left(M=N=2, \delta_{0}=10^{-3}\right)$

\begin{tabular}{|c|c|c|c|c|}
\hline Average SNR & BPSK & QPSK & 16-QAM & 64-QAM \\
\hline 0 & 6.36 & 9.62 & 16.56 & 22.58 \\
\hline 1 & 6.22 & 9.56 & 16.55 & 22.58 \\
\hline 2 & 6.04 & 9.47 & 16.53 & 22.57 \\
\hline 3 & 5.82 & 9.36 & 16.51 & 22.57 \\
\hline 4 & 5.57 & 9.22 & 16.49 & 22.56 \\
\hline 5 & 5.35 & 9.02 & 16.45 & 22.56 \\
\hline 6 & 5.23 & 8.75 & 16.41 & 22.55 \\
\hline 7 & 5.36 & 8.36 & 16.36 & 22.53 \\
\hline 8 & 5.91 & 7.74 & 16.29 & 22.52 \\
\hline 9 & $x$ & 6.62 & 16.20 & 22.50 \\
\hline 10 & $x$ & 1.95 & 16.08 & 22.47 \\
\hline 11 & $x$ & $-\infty$ & 15.92 & 22.44 \\
\hline 12 & $x$ & $-\infty$ & 15.71 & 22.40 \\
\hline 13 & $x$ & $-\infty$ & 15.40 & 22.34 \\
\hline 14 & $x$ & $-\infty$ & 14.95 & 22.27 \\
\hline 15 & $x$ & $-\infty$ & 14.23 & 22.18 \\
\hline 16 & $x$ & $-\infty$ & 12.87 & 22.06 \\
\hline 17 & $x$ & $x$ & $-\infty$ & 21.90 \\
\hline 18 & $x$ & $x$ & $-\infty$ & 21.67 \\
\hline 19 & $x$ & $x$ & $-\infty$ & 21.36 \\
\hline 20 & $x$ & $x$ & $-\infty$ & 20.89 \\
\hline 21 & $x$ & $x$ & $-\infty$ & 20.10 \\
\hline 22 & $x$ & $-\infty$ & 13.55 & 18.36 \\
\hline$\geq 23$ & $x$ & $x$ & $x$ & $-\infty$ \\
\hline
\end{tabular}

of (6) are used to get the switching thresholds for adaptive modulation, and the Huang method means that the constellation specific constants of Table 1 are used. The A-BER approach has SNR gain of 2-3 dB compared to the I-BER method at the same ASE. In the I-BER approach, even though the exact BER function in AWGN channels is used, the A-BER is still below $\delta_{0}$ so that it is too conservative. In the A-BER approach, the optimal method ('H+LM' in the figure), in which constellation specific constants defined in, and Lagrange multiplier are used, gives the best ASE performance while satisfying the A-BER constraint. In case that the constants of (6) are used (' $Z+L M$ '), even though Lagrange multiplier is used, gives lower performance. This means that using an accurate BER function in (13) is important. The sub-optimal method ('H+DS') also gives as good performance as the optimal method. Since the optimal thresholds are not available in high average $\mathrm{SNR}$ range $(\mathrm{ZF}: \geq 33 \mathrm{~dB}$, OSTBC: $\geq 23 \mathrm{~dB}$ ), the last valid thresholds are used (ZF: $32 \mathrm{~dB}$, OSTBC: $22 \mathrm{~dB}$ ) as mentioned in [11]. At low average SNR, the optimal method performs better in terms of ASE than the sub-optimal method. At high average SNR, the suboptimal method is slightly better in terms of ASE than the optimal method because the search of the optimal SNR thresholds fails at high average SNR, and the last valid threshold with a lower SNR was used. Thus, we can use a hybrid scheme which uses the optimal thresholds at low average SNR, and the sub-optimal thresholds at high average SNR.

\section{Performance of the dynamic rate-adaptive MIMO mode switching}

Figures 6 and 7 shows the ASE and A-BER of the proposed scheme. The SNR thresholds of the proposed scheme are obtained from the A-BER approach using the hybrid approach described in the previous section. At low average SNR, the optimal thresholds are used, whereas the sub-optimal thresholds are used at high average SNR where the optimal thresholds cannot be computed (i.e., $\mathrm{ZF}: \geq 33 \mathrm{~dB}$, OSTBC: $\geq 23 \mathrm{~dB}$ ). As shown in Figure 6, the two ASE curves that only one MIMO mode is used cross at about $17 \mathrm{~dB}$. Thus, the mode switching rule of the static method is as follows: if $\rho<17$, the diversity mode (OSTBC) is selected. Otherwise, the spatial multiplexing mode (ZF) is selected. It is observed that the proposed dynamic switching scheme has 1-2 dB SNR gain over the static switching scheme in the $10-26 \mathrm{~dB}$ SNR range while keeping the A-BER close to the target value as shown in Figure 7 . It is also observed in Figure 6 that the proposed mode switching criterion has the same performance as the Demmel condition number (DCN) method. Therefore, the proposed criterion is preferable in terms of computational complexity when the ISE of each MIMO mode is equal to each other.

\section{Conclusion}

In this article, we proposed a dynamic rate-adaptive MIMO mode switching scheme between spatial multiplexing and diversity modes. The proposed dynamic scheme shows better ASE performance than the static MIMO mode switching scheme with adaptive modulation. In the conventional static mode, the MIMO mode changes depending on the average SNR instead of the instantaneous channel condition. We also proposed a suboptimal algorithm to find the SNR thresholds with the ABER constraint in the high average SNR range. When the two MIMO modes have the same ISE, we can use a new mode switching criterion based on the BER exponent. The BER exponent method shows the same performance as the Demmel condition number method with lower computational complexity. Simulations show that the performance of the proposed algorithm is close to that of the optimal case. The proposed algorithms appear to be promising as a practical MIMO mode switching technique. 




Figure 2 ASE performance of the spatial multiplexing system with ZF receiver.

\section{Endnote}

${ }^{a}$ In this article, we use boldface lowercase letters to denote vectors, boldface uppercase to denote matrices. $\mathbf{s}_{k}$ is the $k$ th element of the vector $\mathbf{s}$ while $[\mathbf{H}]_{i, j}$ is the element in the $i$ th row and jth column of the matrix $\mathbf{H} .\|\mathbf{H}\|_{F}$ is the Frobenius norm on $\mathbf{H}$.

${ }^{b}$ We use $(\cdot)^{H}$ for conjugate transpose, and $\mathcal{E}$ to denote expectation.

${ }^{\mathrm{c}} \theta_{L+1}$ is fixed as infinity.

\section{Appendix: Closed form expressions of ASE and} average BER for the two MIMO modes

Spatial multiplexing system with zero-forcing receiver It has been shown in [16] that the pdf of $\gamma_{m, Z F}$ in (2) is distributed as

$$
f\left(\gamma_{m, \mathrm{ZF}}\right)=\frac{M}{\rho(N-M) !}\left(\frac{M \gamma_{m, \mathrm{ZF}}}{\rho}\right)^{N-M} \exp \left(-\frac{M \gamma_{m, \mathrm{ZF}}}{\rho}\right) .
$$

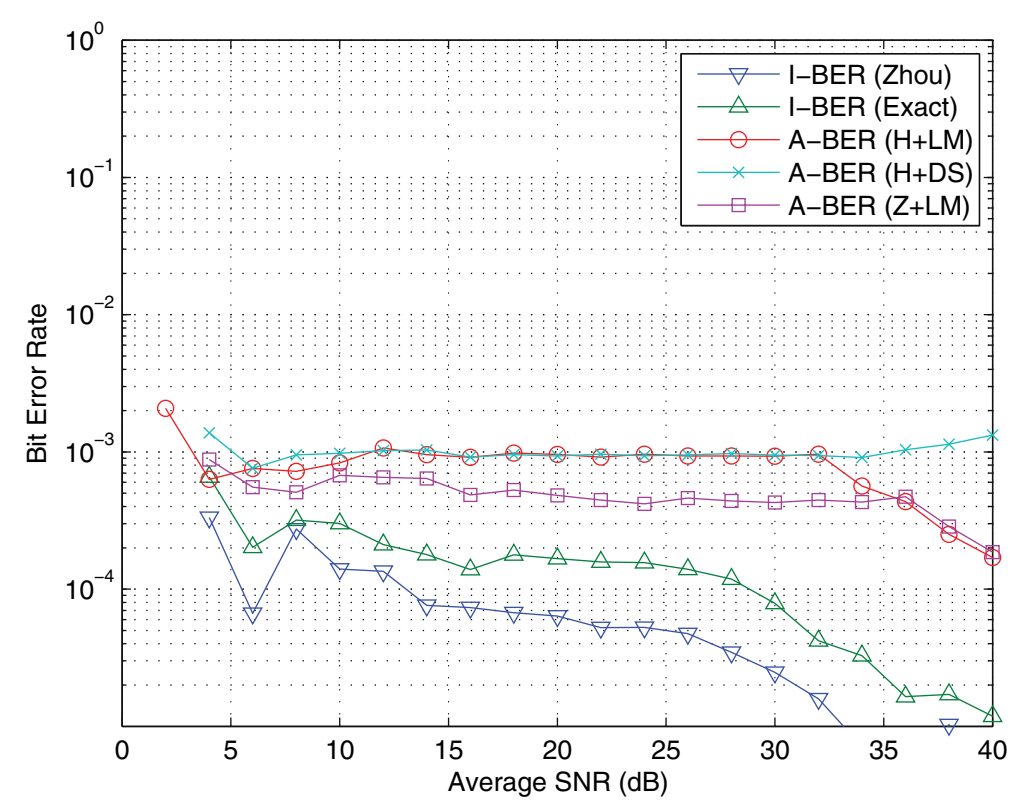

Figure 3 BER performance of the spatial multiplexing system with ZF receiver. 




Figure 4 ASE performance of the OSTBC system.

Defining the set of adjustable thresholds $\Theta_{\mathrm{ZF}}$ as

$$
\Theta_{\mathrm{ZF}}=\left\{\theta_{m, l} \mid m=1, \ldots, M ; l=1, \ldots, L\right\},
$$

the ASE $\eta_{\mathrm{ZF}}$ is given by

$$
\eta_{\mathrm{ZF}}=\sum_{m=1}^{M} \sum_{l=1}^{L} b_{m, l} \int_{\theta_{m, l}}^{\theta_{m, l+1}} f\left(\gamma_{m, \mathrm{ZF}}\right) d \gamma_{m, \mathrm{ZF}}
$$

Since it is assumed that channel is i.i.d., the pdf's of all the $\gamma_{m, \mathrm{ZF}}$ 's are identical.

Thus, dropping the subscript $m$, the ASE can be rewritten as

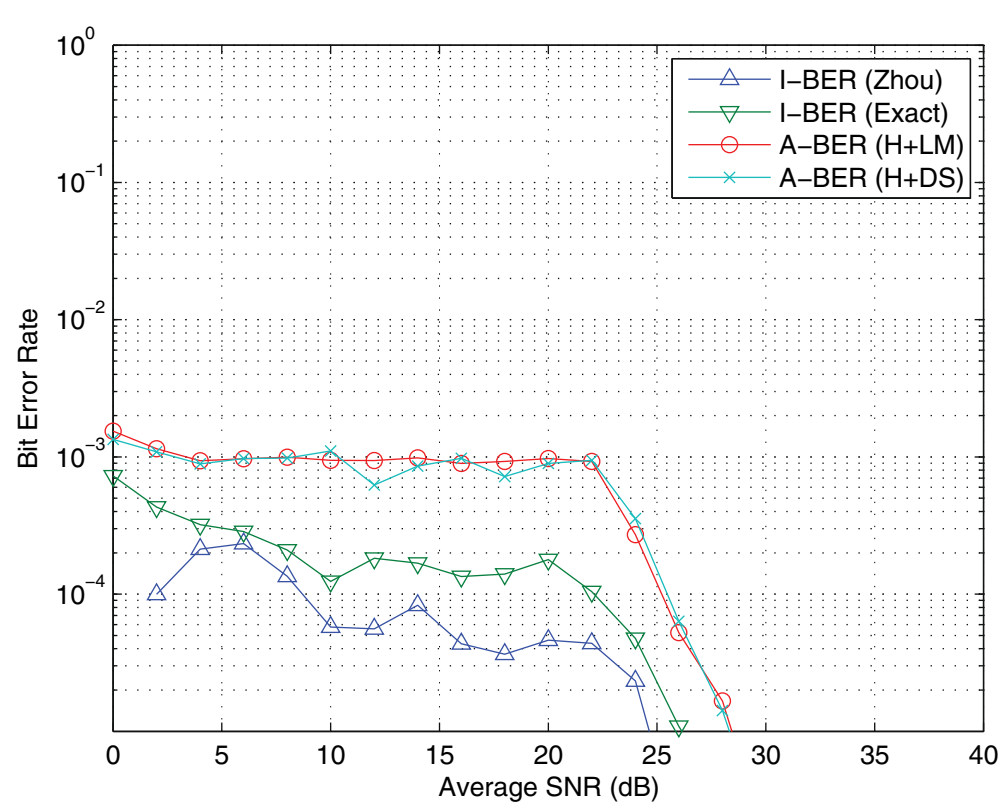

Figure 5 BER performance of the OSTBC system. 




Figure 6 ASE performance of the dynamic rate-adaptive MIMO mode switching system.

Substituting (21) into (24) and using the upper incomplete Gamma function defined as

$$
\Gamma(s, x)=\int_{x}^{\infty} t^{s-1} e^{-t} d t,
$$

$\eta_{\mathrm{ZF}}$ can be expressed as a closed form as follows:

$\eta_{\mathrm{ZF}}=\frac{M}{\Gamma\left(D_{\mathrm{ZF}}\right)} \sum_{l=1}^{L} b_{l}\left\{\Gamma\left(D_{\mathrm{ZF}}, \frac{M}{\rho} \theta_{l}\right)-\Gamma\left(D_{\mathrm{ZF}}, \frac{M}{\rho} \theta_{l+1}\right)\right\}$, where $D_{\mathrm{ZF}}=N-M+1$ and $\Gamma(n)=(n-1)$ !. Likewise, the A-BER $P_{e, \text { avg,ZF }}$ can be denoted as

$$
P_{e, \mathrm{a} g, \mathrm{ZF}}=\frac{M}{\eta_{\mathrm{ZF}}} \sum_{l=1}^{L} b_{l} P_{e, \mathrm{ZF}}(l),
$$

where $P_{e, \mathrm{ZF}}(l)$ is given by

$$
P_{e, \mathrm{ZF}}(l)=\int_{\theta_{l}}^{\theta_{l+1}} P_{e}\left(\gamma_{\mathrm{ZF}}, M_{l}\right) f\left(\gamma_{\mathrm{ZF}}\right) d \gamma_{\mathrm{ZF}}
$$

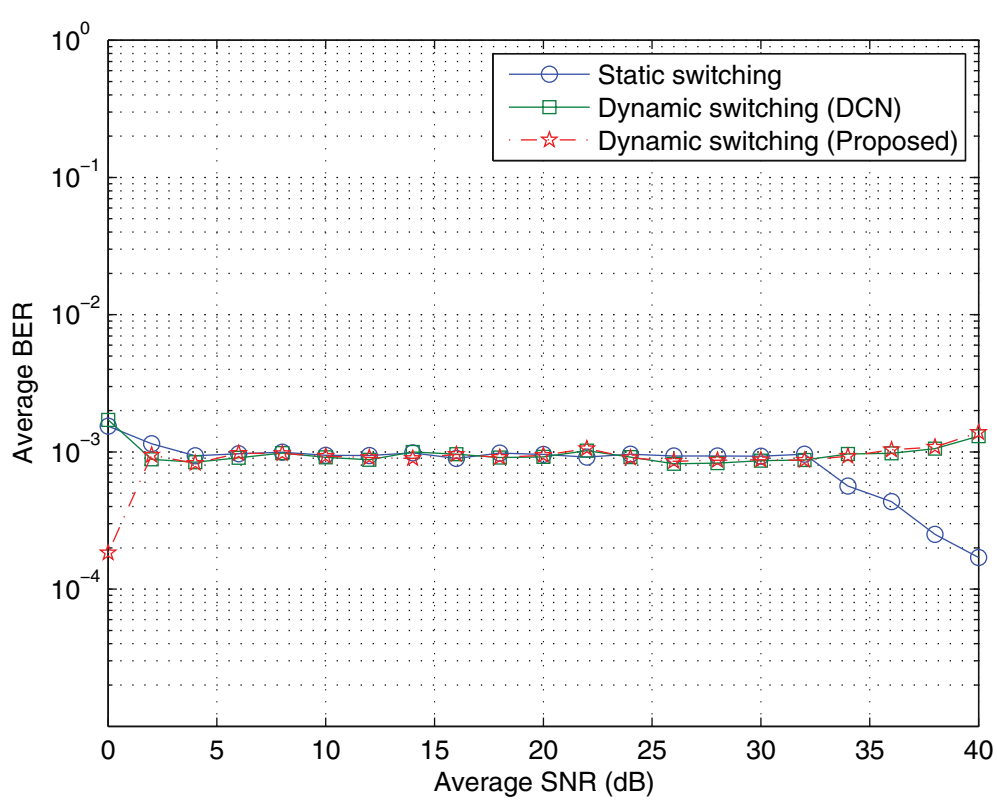

Figure 7 BER performance of the dynamic rate-adaptive MIMO mode switching system. 
Substituting (5) and (21) into (28), $P_{e, \mathrm{ZF}}(l)$ can be expressed as a closed form as follows:

$$
P_{e, \mathrm{ZF}}(l)=\frac{a_{l}\left\{\Gamma\left(D_{\mathrm{ZF}},\left(c_{l}+\frac{M}{\rho}\right) \theta_{l}\right)-\Gamma\left(D_{\mathrm{ZF}},\left(c_{l}+\frac{M}{\rho}\right) \theta_{l+1}\right)\right\}}{\Gamma\left(D_{\mathrm{ZF}}\right)\left(1+\frac{\rho c_{l}}{M}\right)^{D}} .
$$

Substituting (26) and (29) into (27), $P_{e, \text { avg, ZF is finally }}$ obtained as

$$
\begin{aligned}
& P_{e, \mathrm{a} v g, \mathrm{ZF}} \\
& =\frac{\sum_{l=1}^{L} a_{l} b_{l}\left\{\Gamma\left(D_{\mathrm{ZF}},\left(c_{l}+\frac{M}{\rho}\right) \theta_{l}\right)-\Gamma\left(D_{\mathrm{ZF}},\left(c_{l}+\frac{M}{\rho}\right) \theta_{l+1}\right)\right\}}{\left(1+\frac{\rho c_{l}}{M}\right)^{D} \sum_{l=1}^{L} b_{l}\left\{\Gamma\left(D_{\mathrm{ZF}}, \frac{M}{\rho} \theta_{l}\right)-\Gamma\left(D_{\mathrm{ZF}}, \frac{M}{\rho} \theta_{l+1}\right)\right\}} .
\end{aligned}
$$

\section{Orthogonal space-time block coding system}

It is known that the pdf of $\gamma_{\text {OSTBC }}$ in (4) is distributed as [7]

$$
\begin{aligned}
f\left(\gamma_{\text {OSTBC }}\right)= & \frac{\zeta M}{\rho(N M-1) !}\left(\frac{\zeta M \gamma_{\text {OSTBC }}}{\rho}\right)^{N M-1} \\
& \times \exp \left(-\frac{\zeta M \gamma_{\text {OSTBC }}}{\rho}\right) .
\end{aligned}
$$

Since the pdf is also Gamma distributed as that of ZF case, the derivation procedure is similar. Defining the set of adjustable thresholds $\Theta_{\text {OSTBC }}$ as

$$
\Theta_{\mathrm{OSTBC}}=\left\{\theta_{1}, \theta_{2}, \ldots, \theta_{L}\right\},
$$

the ASE $\eta_{\text {OSTBC }}$ is given by

$$
\eta_{\mathrm{OSTBC}}=\zeta \sum_{l=1}^{L} b_{l} \int_{\theta_{l}}^{\theta_{l+1}} f\left(\gamma_{\mathrm{OSTBC}}\right) d \gamma_{\mathrm{OSTBC}} .
$$

Substituting (31) into (33), $\eta_{\text {OSTBC }}$ can be expressed as a closed form as follows:

$$
\eta_{\mathrm{OSTBC}}=\frac{\zeta \sum_{l=1}^{L} b_{l}\left\{\Gamma\left(D_{\mathrm{OSTBC}}, \frac{\zeta M}{\rho} \theta_{l}\right)-\Gamma\left(D_{\mathrm{OSTBC}}, \frac{\zeta M}{\rho} \theta_{l+1}\right)\right\}}{\Gamma\left(D_{\mathrm{OSTBC}}\right)},
$$

where $D_{\text {ОАTBC }}=N M$. Likewise, the A-BER $P_{e, \text { avg, }, \text { ОSTBC }}$ can be denoted as

$$
P_{e, \text { avg }, \text { OSTBC }}=\frac{\zeta}{\eta_{\text {OSTBC }}} \sum_{l=1}^{L} b_{l} P_{e, \text { OSTBC }}(l),
$$

where $P_{e, \text { OSTBC }}(l)$ is given by

$$
P_{e, \text { оттв }}(l)=\int_{\theta_{l}}^{\theta_{l+1}} P_{e}\left(\gamma_{\mathrm{OSTBC}}, M_{l}\right) f\left(\gamma_{\mathrm{osTBC}}\right) d \gamma_{\mathrm{OSTBC}} .
$$

Substituting (5) and (31) into (36), $P_{e, \text { OSTBC }}(l)$ can be expressed as a closed form as follows:

$$
\begin{aligned}
& P_{e, \text { } \text { ОАтв }}(l) \\
& =\frac{a_{l}\left\{\Gamma\left(D_{\text {OSTBC }},\left(c_{l}+\frac{\zeta M}{\rho}\right) \theta_{l}\right)-\Gamma\left(D_{\text {OSTBC }},\left(c_{l}+\frac{\zeta M}{\rho}\right) \theta_{l+1}\right)\right\}}{\Gamma\left(D_{\text {OSTBC }}\right)\left(1+\frac{\rho c_{l}}{\zeta M}\right)^{D_{\text {OSTBC }}}} .
\end{aligned}
$$

Substituting (34) and (37) into (35), $P_{e, \text { avg, О्ттBC }}$ is finally obtained as

$$
\begin{aligned}
& P_{e, \text { avg }, \text { OSTBC }} \\
& =\frac{\sum_{l=1}^{L} a_{l} b_{l}\left\{\Gamma\left(D_{\mathrm{O}},\left(c_{l}+\frac{\zeta M}{\rho}\right) \theta_{l}\right)-\Gamma\left(D_{\mathrm{O}},\left(c_{l}+\frac{\zeta M}{\rho}\right) \theta_{l+1}\right)\right\}}{\left(1+\frac{\rho c_{l}}{\zeta M}\right)^{D_{\mathrm{O}}} \sum_{l=1}^{L} b_{l}\left\{\Gamma\left(D_{\mathrm{O}}, \frac{\zeta M}{\rho} \theta_{l}\right)-\Gamma\left(D_{\mathrm{O}}, \frac{\zeta M}{\rho} \theta_{l+1}\right)\right\}},
\end{aligned}
$$

where $D_{\mathrm{O}}$ means $D_{\mathrm{OSTBC}}$.

\section{Competing interests}

The authors declare that they have no competing interests.

\section{Acknowledgements}

This research was supported in part by Basic Science Research Programs (KRF-2008-314-D00287, 2010-0013397), Mid-career Researcher Program (2010-0027155) through the NRF funded by the MEST, Seoul R\&BD Program (JP091007, 0423-20090051), the KETEP grant (2011T100100151), the INMAC, and BK21.

Received: 17 December 2011 Accepted: 22 June 2012 Published: 31 July 2012

\section{References}

1. GJ Foschini, Layered space-time architecture for wireless communication in a fading environment when using multiple aantennas. Bell Labs. Tech. J. 1(2), 41-59 (1996)

2. SM Alamouti, A simple transmit diversity technique for wireless communications. IEEE J. Sel. Areas Commun. 16(8), 1451-1458 (1998)

3. S Catreux, V Erceg, D Gesbert, RW Heath Jr., Adaptive modulation and MIMO coding for broadband wireless data networks. IEEE Commun. Mag. 40(6), 108-115 (2002)

4. S Zhou, GB Giannakis, Adaptive modulation for multi-antenna transmissions with channel mean feedback. in Proc. IEEE Int. Conf. Commun. (ICC'03) (Anchorage, USA, 2003), pp. 2281-2285

5. A Maaref, S Aïssa, Adaptive modulation using orthogonal STBC in MIMO Nakagami fading channels. in in Proc. IEEE Int. Symp. Spread Spectrum Tech. App. (ISSSTA'04), (Sydney, Australia, 2004), pp. 145-149

6. A Maaref, S Aïssa, Rate-adaptive M-QAM in MIMO diversity systems using space-time block codes. in Proc. IEEE Int. Symp. Personal, Indoor and Mobile Radio Commun. (PIMRC'04), (Barcelona, Spain, 2004), pp. 2294-2298

7. Y Ko, C Tepedelenlioǧlu, Orthogonal space-time block coded rate-adaptive modulation with outdated feedback. IEEE Trans. Wirel. Commun. 5(2), 290-295 (2006)

8. A Müller, J Speidel, Adaptive modulation for MIMO spatial multiplexing systems with zero-forcing receivers in semi-correlated Rayleigh fading channels. in Proc. Int. Wireless Commun. and Mobile Computing Conf. (IWCMC'06), (Vancouver, Canada, 2006), pp. 665-670

9. J Huang, S Signell, Discrete rate spectral efficiency improvement by scheme switching for MIMO systems. in Proc. IEEE Int. Conf. Commun (ICC'08), (Beijing, China, 2008), pp. 3998-4002

10. J Huang, S Signell, On spectral efficiency of low-complexity adaptive MIMO systems in Rayleigh fading channel. IEEE Trans. Wirel. Commun. 8(9), 4369-4374 (2009) 
11. J Huang, S Signell, On performance of adaptive modulation in MIMO systems using orthogonal space-time block codes. IEEE Trans. Veh. Technol. 58(8), 4238-4247 (2009)

12. RW Heath Jr., AJ Paulraj, Switching between diversity and multiplexing in MIMO systems. IEEE Trans. Commun. 53(6), 962-968 (2005)

13. A Paulraj, R Nabar, D Gore, Introduction to Space-Time Wireless Communications (Cambridge University Press, New York, 2008)

14. MP Fitz, JP Seymour, On the bit error probability of QAM modulation. Int J. Wirel. Inf. Netws. 1(2), 131-139 (1994)

15. P Li, D Paul, R Narasimhan, J Cioffi, On the distribution of SINR for the MMSE MIMO receiver and performance analysis. IEEE Trans. Inf. Theory. 52(1), 271-286 (2006)

16. D Gore, RW Heath Jr., A Paulraj, On performance of the zero forcing receiver in presence of transmit correlation. in Proc. IEEE Int. Symp. Inf. Theory (ISIT'02) (Lausanne, Switzerland, 2002), p. 159

\section{doi:10.1186/1687-1499-2012-238}

Cite this article as: Kim and Lee: Dynamic rate-adaptive MIMO mode switching between spatial multiplexing and diversity. EURASIP Journal on Wireless Communications and Networking 2012 2012:238.

\section{Submit your manuscript to a SpringerOpen ${ }^{\mathcal{O}}$ journal and benefit from:}

- Convenient online submission

- Rigorous peer review

- Immediate publication on acceptance

- Open access: articles freely available online

- High visibility within the field

- Retaining the copyright to your article

Submit your next manuscript at $\boldsymbol{\nabla}$ springeropen.com 